\title{
DOE/ER/25/05-T2
}

\section{Enhancing Functionality and Performance in the PVM Network Computing System}

\author{
U.S. Department of Energy Grant DE-FG05-91ER25105 (A004) \\ Period 2 Progress Report \& Request for Continuation \\ Vaidy Sunderam \\ Department of Mathematics and Computer Science \\ Emory University, Atlanta, GA 30322, USA \\ vss@mathcs.emory.edu
}

\section{Overview}

The research funded by this grant is part of an ongoing research project in heterogeneous distributed computing with the PVM system, at Emory as well as at Oak Ridge Labs and the University of Tennessee. This grant primarily supports research at Emory that continues to evolve new concepts and systems in distributed computing, but it also includes the PI's ongoing interaction with the other groups in terms of collaborative research as well as software systems development and maintenance. We have continued our second year efforts (July 1995 - June 1996), on the same topics as during the first year, namely a) visualization of PVM programs to complement XPVM displays; (b) I/O and generalized distributed computing in PVM; and (c) evolution of a multithreaded concurrent computing model.

\section{Overall Progress}

Progress has been made in each of the three areas of focus, in the initial project period and is described below:

\subsection{PVM RPC and PIOUS}

To expand the scope and applicability of the PVM system, we have developed an alternate interface to PVM that supports client-server computing. The core of this interface is a 


\section{DISCLAIMER}

Portions of this document may be illegible in electronic image products. Images are produced from the best available original document. 
remote procedure call (RPC) facility that permits the specification and export of services which may be invoked by clients using the well-established RPC paradigm and mechanics. Previous experiences have established that this model is natural and effective for programming distributed applications, and that RPC can be implemented in message-passing systems like PVM with a minimum of overhead. This model also enables significant additional functionality such as user-transparent load balancing, failure resilience, and adaptive parallelism. A preliminary implementation of the RPC system has been completed and both functionality and performance have been found to be significantly improved and useful in preliminary experiments.

The PIOUS project has been ongoing for two years now, and along with RPC support, is the basis for extending PVM applicability to data bound parallel programs. The PIOUS project has progressed very well, with the development and release of beta level software and libraries, which have attained high levels of scalable I/O performance over networks and clusters of workstations. The PIOUS architecture was refined during this project period and the PVM implementation of PIOUS was completed. In order to provide PVM applications with a uniform filesystem interface, PIOUS employs data declustering to exploit the combined file $\mathrm{I} / \mathrm{O}$ and buffer cache capacities of networked computing resources, and transaction-based concurrency control, to guarantee access consistency without explicit synchronization. PIOUS consists of a set of data servers, a service coordinator, and library routines linked with client processes. An underlying transport mechanism is assumed to carry messages between client processes and components of the PIOUS architecture. A prototype of the PIOUS system has been developed and has been distributed to the PVM community. Further, a student Nancy Sedora, has used the PIOUS system to implement the NHT-1 I/O benchmarks over PVM and measure I/O throughput - we have found that upwards of $80 \%$ of the transport bandwidth delivered by PVM was available to the I/O subsystem, despite the concurrency control synchronization and the transaction based I/O mechanism used.

\subsection{Threads-based computing with TPVM}

The TPVM (Threads-oriented PVM) system is an experimental auxiliary subsystem for the PVM distributed system, which supports the use of lightweight processes or "threads" as the basic unit of parallelism and scheduling. TPVM provides a library interface which presents both a traditional, task based, explicit message passing model, as well as a datadriven scheduling model that enables straightforward specification of computation based on data dependencies. The TPVM system comprises three basic modules: a library interface that provides access to thread-based distributed concurrent computing facilities, a portable thread interface module which abstracts the required threads-related services, and a thread server module which performs scheduling and system data management. Our design is still under development, but a prototype implementation has allowed us to perform a number of preliminary experiments. In this phase of the project, we have successfully refined the 
TPVM implementation and design, and have ported it to multiple platforms. Results from its use are very encouraging and numerous external users have retrieved and are using the software. We are also in the process of converting the NAS parallel benchmark suite to use TPVM and have had some success in reducing latency and achieving increased communication-computation overlap.

\subsection{PVaniM}

The PVaniM 2.0 system provides online and postmortem visualization support as well as rudimentary I/O for long running, communication-intensive PVM applications. PVaniM 2.0 provides these features while using several techniques to keep system perturbation to a minimum. The online graphical views provided by PVaniM 2.0 provide insight into message communication patterns, the amount of messages and bytes sent, host utilization, memory utilization, and host load information. For online visualization analysis, PVaniM 2.0 utilizes sampling to gather data regarding interesting aspects of the application as well as interesting aspects of the cluster environment. With sampling, necessary statistics are collected and sent to the monitor intermittently. The rate at which the application data is sent to the monitor is a parameter that may be set by the user. With lower sampling rates, the application will experience less perturbation, but the graphical views will not be updated as frequently. For offline visualization analysis, PVaniM 2.0 utilizes buffered tracing to provide support for fine-grain visualization systems. Currently, PVaniM produces tracefiles for the prototype PVaniM system. A converter is provided that allows the user to also use the popular ParaGraph system using techniques similar to PGPVM. This system has been found to be a very useful complement to XPVM and provides extremely useful facilities for PVM developers. Over 200 copies of the software have been distributed and feedback from users is very positive.

\section{Research Output}

\subsection{Invited Talks and Professional Activities}

1. "An RPC Facility for PVM", invited talk, High Performance Computing and Networking, HPCN'96, Brussels, April 1996.

2. "PVM Data Transfers on SCI Workstation Clusters: Early Experiences", contributed talk, 4th PVM User's Group Meeting, Santa Fe, NM, February 1996.

3. "Performance and Functionality Issues in Heterogeneous Systems", invited talk, Dartmouth College, Hanover, NH, November 1995.

4. "Recent Developments and Future Trends in Heterogeneous Concurrent Computing", invited talk, University of Fribourg, Fribourg, Switzerland, July 1995. 
5. "Concurrent Computing with PVM", invited workshop, Technical University of Munich, Munich, Germany, June 1995.

\subsection{Publications:}

1. S. Moyer and V. S. Sunderam, "Characterizing Concurrency Control Performance for the PIOUS Parallel File System", Journal of Parallel and Distributed Computing, to appear, 1996.

2. B. Topol, J. Stasko, and V. S. Sunderam, "The Dual Timestamping Methodology for Visualizing Distributed Applications", IEEE Parallel and Distributed Technology, to appear, 1996.

3. V.S.Sunderam and S. Moyer, "Parallel I/O for Distributed Systems: Issues and Implementation", Future Generation Computer Systems, to appear, May 1996.

4. A. Ferrari and V. S. Sunderam, "Multi-Paradigm Distributed Computing with TPVM", submitted to Journal of Concurrency: Practice and Experience, March 1996.

5. B. Topol, J. Stasko, and V. S. Sunderam, "Monitoring and Visualization in Cluster Environments", submitted to Journal of Parallel and Distributed Computing, March 1996.

6. A. Zadroga, A. Krantz, S. Chodrow, V. Sunderam, "An RPC Facility for PVM", Proceedings - High-Performance Computing and Networking '96, Brussels, Belgium, Springer-Verlag, pp. 798-805, April 1996.

7. V. S. Sunderam and H. Hellwagner, "Parallel Distributed Computing on SCI Workstation Clusters: Early Experiences", Proceedings - Parallele Algorithmen, Rechnerstrukturen und Systemsoftware Workshop Stuttgart, Germany, pp. 27-34, October 1995. 


\section{Forthcoming Project Period Plans}

During the third project period, we intend to continue to develop fully the "generalized distributed computing" subsystems, i.e. the RPC/coordination model and I/O, and continue further investigations and developments on the threads framework, TPVM.

\subsection{Coordination, $\mathrm{RPC}$, and $\mathrm{I} / \mathrm{O}$}

The PVM RPC facility that was developed has demonstrated that message-passing systems such as PVM can very effectively support client server computing. This is an important advantage for many distributed computing applications such as remote intsrument controllers, transaction processors, and software agents. We believe that an effective infrastructure to support such applications will be extremely valuable and can utilize the existing message passing and process management infrastructure in PVM to great advantage. We will therefore extend the (RPC system of) PVM to support the coordination model for distributed systems. Essentially, the coordination models permits unrelated processes or computational entities to cooperate to solve a given problem. This both implies, and is enabled by, decoupling processes and their interactions in space and in time. Implementations of such models typically employ a global name space and bulletin board or mailbox facilities for exchanging data. We will investigate possible methods and programming interfaces for supporting the coordination model within PVM and will design and implement such a system. Simultaneously we will also further refine and optimize the more well-known and well-established RPC paradigm for PVM and release this subsystem for external use. We will also continue to work on the PIOUS I/O subsystem, focusing on techniques for data layout and imposing file structure semantics on parallel files.

\subsection{TPVM for High Performance Scientific Computing}

Our preliminary experiences with a threads-based computing model have demonstrated that the most serious obstacle to efficient network computing, namely latency, can be addressed effectively by overlapping computation and communication using light weight processes. However, it is also clear that this can be achieved only in cases where parallel algorithms are not loosely synchronous, the way most SPMD numerical applications tend to be. Therefore, in order to fully exploit the potential of multithreaded network computing, staggered partitioning and scheduling schemes will be investigated. We will attempt to design such strategies that can be applied to existing SPMD codes, and will test our ideas on the NAS parallel benchmarking suite. Simultaneously, we will also explore an extension to the idea of threads-based computing, using Java or Perl scripts to encapsulate problem pieces and accompanying data, and transport them to optimal machines in the PVM hostpool, for execution. We have already experimented with a Java extension to PVM, and this approach, coupled with multithreaded computing models, appears to be very promising. 


\section{DISCLAIMER}

This report was prepared as an account of work sponsored by an agency of the United States Government. Neither the United States Government nor any agency thereof, nor any of their employees, makes any warranty, express or implied, or assumes any legal liability or responsibility for the accuracy, completeness, or usefulness of any information, apparatus, product, or process disclosed, or represents that its use would not infringe privately owned rights. Reference herein to any specific commercial product, process, or service by trade name, trademark, manufacturer, or otherwise does not necessarily constitute or imply its endorsement, recommendation, or favoring by the United States Government or any agency thereof. The views and opinions of authors expressed herein do not necessarily state or reflect those of the United States Government or any agency thereof. 